Evaluation of Salt Tolerance at Germination Stage in Cowpea [Vigna unguiculata (L.) Walp]

\author{
Waltram Second Ravelombola, Ainong Shi ${ }^{1}$, Yuejin Weng, \\ and John Clark \\ Department of Horticulture, 316 PTSC, University of Arkansas, Fayetteville, \\ AR 72701
}

Dennis Motes
Vegetable Research Center, University of Arkansas, Alma, AR 72921

\title{
Pengyin Chen
}

University of Missouri - Fisher Delta Research Center, 147 State Hwy T, Portageville, MO 63873

\section{Vibha Srivastava \\ Department of Crop, Soil, and Environmental Sciences, 215 PTSC, University of Arkansas, Fayetteville, AR 72701}

Additional index words. Vigna unguiculata, breeding, germplasm, salinity, screening

\begin{abstract}
Cowpea is a leguminous and versatile crop which provides nutritional food for human consumption. However, salinity unfavorably reduces cowpea seed germination, thus significantly decreasing cowpea production. Little has been done for evaluating and developing salt-tolerant cowpea genotypes at germination stage. The objectives of this research were to evaluate the response of cowpea genotypes to salinity stress through seed germination rate and to select salt-tolerant cowpea genotypes. The seed germination rates under nonsalt condition and salinity stress (150 mM NaCl) were evaluated in 151 cowpea genotypes. Four parameters, absolute decrease (AD), the inhibition index (II), the relative salt tolerance (RST), and the salt tolerance index (STI) were used to measure salt tolerance in cowpea. The results showed that there were significant differences among the 151 cowpea genotypes for all parameters $(P$ values $<0.0001)$. The AD in germination rate was $5.8 \%$ to $94.2 \%$; the II varied from $7.7 \%$ to $100 \%$; the RST ranged from 0 to 0.92 ; and STI varied from 0 to 0.92 . A high broad sense heritability $\left(\mathrm{H}^{2}\right)$ was observed for all four parameters. High correlation coefficients $(r)$ were estimated among the four parameters. PI582422, 09-529, PI293584, and PI582570 were highly salt tolerant at germination stage. In addition, genotypes from the Caribbean and Southern Asia exhibited better tolerance to salinity, whereas those from Europe and North America were the most saltsusceptible.
\end{abstract}

Cowpea [Vigna unguiculata (L.) Walp] $(2 n=2 x=22)$ is a legume of economic importance worldwide. It is widely grown in Central and West Africa with a production estimated at 5.4 million tons of dry seed (Olufajo, 2012) and also grown in Latin America, Southeast Asia, and in the United States (Muchero et al., 2009; Tan et al., 2012). Cowpea plays an essential role for food security and ecosystem services. Cowpea has high seed protein content and other nutritional components for human benefit. It also supplies nitrogen to soils (Duke, 1990). Mamiro et al., (2011) reported that cowpea dried seeds had a fat concentration ranging from $5.4 \%$ to $11.2 \%$ depending on cultivar. The calcium content ranged from 360 to $992.4 \mathrm{mg} / \mathrm{kg}$. The concentration of zinc

Received for publication 9 June 2017. Accepted for publication 11 July 2017.

${ }^{1}$ Corresponding author. E-mail: ashi@uark.edu. varied between 31.5 and $35.6 \mathrm{mg} / \mathrm{kg}$. The iron content ranged from 27.6 to $28.9 \mathrm{mg} / \mathrm{kg}$. In addition, their results suggested that cowpea leaves were also rich in micronutrients. In the United States, cowpea, also commonly referred as southernpea, blackeye pea, crowder pea, lubia, niebe, coupe, or frijole is usually grown by small-scale farmers (less than 50 ha) mainly in Southern States, and there is a significant blackeye bean industry in California. It is a profitable crop for growers (Davis et al., 2012; Okiror et al., 2008).

Germination is a pivotal step for crop propagation. Studies reported that germination is highly influenced by a large number of environmental factors. Mistura et al., (2011) stated that salinity affected germination of legumes and plant vigor. Dutta and Bera (2014) found a noticeable decrease in seed germination, plant growth, and vigor indices for mung bean supplied with salt treatment. In addition, Zhang et al., (2013) stated that low temperatures undermine cowpea germination. Their results indicated that germination rate decreased to $18 \%$ at $10{ }^{\circ} \mathrm{C}$. Reddy and Reddy (2016) reported that excessive and incessant rainfall decreased the seed germination of soybean in India. Low germination unfavorably affects crop production. Germination ensures the propagation of plants to properly ensure food supplies for human consumption (Bewley, 2003). Moreover, seed quality is an important feature required by seed testing centers. To ensure a stable market, seed producer companies have to provide good quality seeds exhibiting high germination rate.

Saline soil is characterized by high concentration of cations $\left(\mathrm{K}^{+}, \mathrm{Mg}^{2+}, \mathrm{Ca}^{2+}\right.$, and $\left.\mathrm{Na}^{+}\right)$ and anions $\left(\mathrm{NO}_{3}{ }^{-}, \mathrm{HCO}_{3}^{-}, \mathrm{SO}_{4}{ }^{2-}\right.$, and $\left.\mathrm{Cl}^{-}\right)$ (Wallender and Tanji, 2011). Sodium chloride is the most well-known salt causing damage in agriculture (Ayers and Westcot, 1985). In addition, Shannon (1997) reported that the concentration of salt in cultivated areas has been increasing because of inappropriate irrigation, fertilization, or other factors. Such increase is estimated at 1 to 60 t/ha annually. Läuchli and Lüttge (2002) pointed out that costs related to salinity issue were 12 billon US dollars per year. Rock weathering, seawater, rain, deforestation, air pollution, and contamination of river waters by chemical and domestic animals are substantial sources of salinity in agriculture (Omami and Hammes, 2006; Rengasamy et al., 2006).

However, little has been done regarding studies on seed germination in cowpea along with the factors that markedly affect germination rate. A study conducted by Zahedi et al., (2012) reported that salinity decreased germination rate in cowpea, and poor seed germination resulted in a significant reduction in yield. In addition, salinity had negative effects on plant growth, cell extension, cell division, and photosynthesis (Maas and Hoffman, 1977).

The evaluation of salt tolerance requires a controlled environment. Field screening is an available technique to screen plant genotypes for salinity. For this method, seeds are sown directly in soil with high salt concentration. However, because of the variability of salt content in the field and the considerable influence of other factors such as moisture content in soil, soil fertility, temperature, light, transpiration, and weather, the results might be highly biased and nonconclusive in a field setting (Parker et al., 1983; Pathan et al., 2007; Yang and Blanchar, 1993). Plant genotypes can be also hydroponically screened by using nutrient solution as a medium growth and adding salt solution after germination and establishment (An et al., 2001). However, such a method is expensive. Therefore, evaluating salt tolerance at germination stage would be of interest because doing so will add information to genotype response at different stages of plant growth and development

Screening for salt-tolerant genotypes at germination stage is one of the most cost 
effective ways to tackle salinity-related issues. In this view, some research has dealt particularly with the effects of salinity on germination rate and emergence (Badia and Meiri, 1994; Carter et al., 2006; Kent and Lauchli, 1985; Mauromicale and Licandro, 2002). Kan et al. (2015) evaluated the effects of salt stress on a panel of 191 soybean (Glycine $\max$ L.) genotypes and identified eight SNP markers highly associated with tolerance to salinity at germination. Concerning cowpea, Wests and Francois (1982) suggested that a salt concentration higher than $120 \mathrm{~mm}$ would reduce cowpea germination. Salinity engenders osmotic or toxicity effects, which result in low seed germination (Waisel, 1972). In addition, Taffouo et al. (2009) reported that high sodium chloride concentration significantly affected germination rate in cowpea. Ashebir et al. (2013) studied the effects of salinity at germination in cowpea. Their results revealed that there was a significant variability in response to salt stress among cowpea genotypes. They found that the genotypes '211557' and 'Asebot' were salt tolerant at germination stage. The objectives of this study were to evaluate the responses of cowpea genotypes to salinity $(\mathrm{NaCl})$ at germination stage, to screen salttolerant cowpea genotypes, and to select for the most accurate parameter for assessing cowpea salt tolerance at germination stage for a salt-tolerance breeding program.

\section{Materials and Methods}

Plant materials. A total of 151 cowpea genotypes consisting of 116 United States Department of Agriculture (USDA) Germplasm Resources Information Network (GRIN) germplasm accessions and 35 University of Arkansas lines were used in this study (Table 1). The 116 germplasm accessions were originally collected from 31 countries and classified into 12 regions depending on their origin (Caribbean, Eastern Africa, Southern Africa, Western Africa, Eastern Asia, Southeastern Asia, Southern Asia, Western Asia Europe, Latin America, and North America). All original seeds of the GRIN germplasm were obtained from USDA Plant Genetic Resources Conservation Unit at Griffin, GA, and were increased at the Research and Extension Station of University of Arkansas at Fayetteville, AR, in the summers 2014 and 2015.

Determination of optimal seed germination temperature and salt concentration. Two pilot experiments were conducted to determine the optimal temperature and salt $(\mathrm{NaCl})$ concentration for cowpea seed germination. For the temperature experiment, the cowpea cultivar Early Scarlet was used to test seed germination rates under three thermogradient temperatures $\left(25,28\right.$, and $\left.31^{\circ} \mathrm{C}\right)$. The results showed that 'Early Scarlet' had the highest seed germination rate at $28{ }^{\circ} \mathrm{C}$, which was the same optimum temperature for cowpea seed germination reported by Souza et al., (2004). In regard to salt concentration, five cowpea genotypes ('Early
Scarlet', '07-303', '09-655', 'PI293584', and 'PI527561') were tested for seed germination under six levels of concentrations $(0$, $50,100,150,200$, and $250 \mathrm{~mm} \mathrm{NaCl}$ ) to determine the optimal $\mathrm{NaCl}$ concentration for salt stress. The concentrations were obtained by dissolving at 2.92, 5.84, 8.77, 11.69 , and $14.61 \mathrm{~g}$ of sodium chloride powder of Science Company ${ }^{\circledR}$, Lakewood, $\mathrm{CO}$, into $1 \mathrm{~L}$ of distilled water, respectively. The salt concentration where germination rate between cowpea genotypes was the most significantly different was chosen as the optimal salt concentration to evaluate salt tolerance at germination stage.

Germination conditions. The cowpea seeds used in this study were harvested from the field of University of Arkansas Research and Extension Center at Fayetteville, AR, during Summer 2015. Seeds having uniform size from each cowpea genotype were selected. To avoid any contamination, clean seeds were selected, and petri dishes used for germination were sterilized $60 \mathrm{~s}$ by washing with bleach $2 \%$ followed by $75 \%$ ethanol.

Forty seeds from each cowpea genotype were put on paper filter (Laboratory Nerd) (Avogadro's Laboratory Supply, Inc, Shamong NJ 08088), which was previously placed in a petri dish of $9 \mathrm{~cm}$ in diameter. The treatment consisted of adding $14 \mathrm{~mL}$ of $\mathrm{NaCl}$ solution and deionized water for the control $(0 \mathrm{~mm} \mathrm{NaCl})$ to each dish. After treatments were applied, the petri dishes were placed in an incubator New Brunswick Scientific Innova $4230^{\circledR}$ (Manasquan, $\mathrm{NJ}$ ) at $28{ }^{\circ} \mathrm{C}$ for $48 \mathrm{~h}$. Each genotype and salt treatment combination was placed on three different shelves in the incubator with three replicates, and each shelf was treated as a block. The experiment was run in multiple times because of space limitations. After each run, $75 \%$ ethanol solution was sprayed into the incubator to limit any microbial growth.

Measurements. The seed germination data were gathered in $48 \mathrm{~h}$ after placing the petri dishes in the incubator. The seed germination rate was recorded when the radicle reached one third of the seed length. The performance of the cowpea genotype under salinity stress was evaluated by computing the values of AD due to salinity, II (González, 1996), RST, and STI (Fernandez, 1992; Saad et al., 2014). These parameters were estimated using the following formulas:

$$
\begin{gathered}
\text { Absolute Decrease }(\mathrm{AD})=\mathrm{GC}-\mathrm{GS} ; \\
\text { Inhibition Index }(\mathrm{II})=100 *(\mathrm{GC}-\mathrm{GS}) /(\mathrm{GC}) ; \\
\text { Relative Salt Tolerance }(\mathrm{RST})=\mathrm{GS} / \mathrm{GC} ;
\end{gathered}
$$$$
\text { Salt Tolerance Index }(\mathrm{STI})=(\mathrm{GS} * \mathrm{GC}) /\left(\mathrm{GC}_{\mathrm{av}}\right)^{2} \text {, }
$$

where $\mathrm{GC}=$ seed germination rate without salt stress, GS = seed germination under salt stress, and $\mathrm{GC}_{\mathrm{av}}=$ average of the seed germination rate of a cowpea genotype without salt stress.

Experimental design. Regarding the preliminary test related to the determination of the optimal salt $(\mathrm{NaCl})$ concentration, a two-factor factorial (genotype $\mathrm{X}$ salt) organized in a randomized complete block design (RCBD) with three blocks was adopted. The genotype consisted of 'Early Scarlet', '07-303', '09-655', 'PI293584', and 'PI527561', and the salt treatment levels were $0,50,100,150,200$, and $250 \mathrm{~mm}$. Three replications per genotype and salt treatment combination were used.

With respect to the assessment of salt tolerance among the 151 cowpea genotypes, the design was similar to that of the preliminary test using RCBD. However, the salt treatments were the optimal salt concentration from the preliminary test $(150 \mathrm{~mm} \mathrm{NaCl})$ and the deionized water with $0 \mathrm{~mm} \mathrm{NaCl}$. Each salt-genotype combination was assigned to petri dishes, replicated three times, and each replication corresponded to each of the three shelves in the incubator and the shelf was used as a block.

Data analysis. The parameters used for the analysis resulted from pairing data on a genotype under salinity treatment and without salt stress. Therefore, the statistical model for the analysis was as described below.

In the calculations, $Y_{\mathrm{ijk}}=$ value of the parameters for the $j$ th cowpea genotype on the $i$ th shelve at the $k$ th replication, for $i=1$, 2,$3 ; j=1, \ldots, 151$, and $k=1,2,3$.

$$
Y_{\mathrm{ijk}}=\mu+S_{\mathrm{i}}+G_{\mathrm{j}}+\varepsilon_{\mathrm{ijk}}
$$

where $\mu=$ constant (overall mean), $S_{\mathrm{i}}=$ effect of the $i$ th shelf (random effect) on the variability of the response, $G_{\mathrm{j}}=$ effect of the $j$ th genotype (fixed effect) on the mean response, and $\boldsymbol{\varepsilon}_{\mathrm{ijk}}=$ experimental error associated with the ijkth observation. In this study, the effects of experiment runs would be assumed as negligible as the germination study was carried out in an incubator. However, the effect of shelves in the incubator should be taken into account because there could be within incubator temperature variability.

The analysis of variance (ANOVA) test was carried out using the general linear model (GLM) procedure of JMP Genomics 7 (SAS Institute, Cary, NC). The mean separation was performed using the Student's $t$ test at alpha $=0.05$. The descriptive statistics were generated using "Tabulate"; the correlations among the parameters were analyzed using "Multivariate Methods" by "Multivariate" function; and the distributions of the data were drawn using "Distribution" in JMP Genomics 7 .

The broad sense heritability $\left(\mathrm{H}^{2}\right)$ was obtained from (Hosseini et al., 2012)

$$
\begin{aligned}
\mathrm{H}^{2} & =\left(\sigma_{\mathrm{G}}^{2} / \sigma_{\mathrm{P}}^{2}\right) \times 100 \\
& =\left[\sigma_{\mathrm{G}}^{2} /\left(\sigma_{\mathrm{G}}^{2}+\left(\sigma_{\mathrm{E}}^{2} / \mathrm{r}\right)+\left(\sigma_{\mathrm{S}}^{2} / \mathrm{s}\right)\right] \times 100\right.
\end{aligned}
$$

where $\sigma_{\mathrm{G}}^{2}=$ genotypic variance, $\sigma_{\mathrm{P}}^{2}=$ phenotypic variance, $\sigma_{\mathrm{E}}^{2}=$ variance associated with the experimental error, $\sigma_{\mathrm{S}}^{2}=$ variance associated with the shelf, $s=$ number of shelves, and $r=$ number of replications per treatment. 


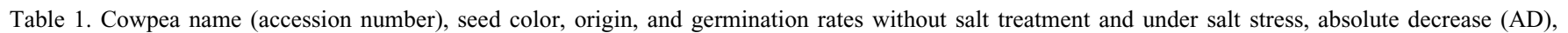
inhibition index (II), relative salt tolerance (RST), and salt tolerance index (STI).

\begin{tabular}{|c|c|c|c|c|c|c|c|c|}
\hline Accession number & Seed color & Origin & Nonstress $(\%)$ & Stress $(\%)$ & AD_(\%) & II_(\%) & RST & STI \\
\hline$\overline{07-303}$ & Red & AR, USA & 82.50 & 60.83 & 21.67 & 25.83 & 0.74 & 0.73 \\
\hline $09-105$ & Cream & AR, USA & 89.17 & 46.67 & 42.50 & 47.59 & 0.52 & 0.52 \\
\hline 09-1090 & Pinkeye & AR, USA & 85.00 & 35.00 & 50.00 & 58.85 & 0.41 & 0.41 \\
\hline $09-181$ & Pinkeye & AR, USA & 72.50 & 49.17 & 23.33 & 32.09 & 0.68 & 0.68 \\
\hline $09-231$ & Pinkeye & AR, USA & 68.50 & 42.50 & 26.00 & 37.65 & 0.62 & 0.62 \\
\hline $09-239$ & Pinkeye & AR, USA & 80.83 & 34.17 & 46.67 & 57.88 & 0.42 & 0.43 \\
\hline $09-268$ & Cream & AR, USA & 83.33 & 16.67 & 66.67 & 79.92 & 0.20 & 0.20 \\
\hline $09-295$ & Pinkeye & AR, USA & 64.17 & 35.83 & 28.33 & 44.12 & 0.56 & 0.56 \\
\hline $09-307$ & Blackeye & AR, USA & 96.67 & 46.67 & 50.00 & 51.71 & 0.48 & 0.48 \\
\hline $09-316$ & Black Holstein & AR, USA & 90.00 & 31.67 & 58.33 & 64.75 & 0.35 & 0.35 \\
\hline $09-323$ & Pinkeye & AR, USA & 86.67 & 50.00 & 36.67 & 42.05 & 0.58 & 0.58 \\
\hline $09-393$ & Pinkeye & AR, USA & 87.50 & 0.83 & 86.67 & 99.02 & 0.01 & 0.01 \\
\hline $09-452$ & Black Holstein & AR, USA & 88.33 & 11.67 & 76.67 & 86.76 & 0.13 & 0.13 \\
\hline $09-455$ & Blackeye & AR, USA & 77.50 & 57.50 & 20.00 & 25.89 & 0.74 & 0.75 \\
\hline $09-470$ & Pinkeye & AR, USA & 91.67 & 44.17 & 47.50 & 51.61 & 0.48 & 0.48 \\
\hline $09-529$ & Blackeye & AR, USA & 85.50 & 75.00 & 10.50 & 12.16 & 0.88 & 0.88 \\
\hline $09-655$ & Pinkeye & AR, USA & 92.50 & 28.33 & 64.17 & 69.01 & 0.31 & 0.30 \\
\hline $09-686$ & Pinkeye & AR, USA & 93.33 & 37.50 & 55.83 & 59.80 & 0.40 & 0.40 \\
\hline $09-692$ & Pinkeye & AR, USA & 92.50 & 39.17 & 53.33 & 57.33 & 0.43 & 0.42 \\
\hline $09-697$ & Pinkeye & AR, USA & 68.33 & 32.50 & 35.83 & 52.26 & 0.48 & 0.47 \\
\hline $09-714$ & Pinkeye & AR, USA & 80.83 & 40.00 & 40.83 & 50.47 & 0.50 & 0.50 \\
\hline $09-741$ & Red Holstein & AR, USA & 87.50 & 65.83 & 21.67 & 24.41 & 0.76 & 0.75 \\
\hline $09-744$ & Cream & AR, USA & 85.83 & 42.50 & 43.33 & 50.40 & 0.50 & 0.49 \\
\hline $09-745$ & Red Holstein & AR, USA & 89.17 & 6.67 & 82.50 & 92.49 & 0.08 & 0.07 \\
\hline 09-749 & Red Holstein & AR, USA & 76.67 & 27.50 & 49.17 & 64.21 & 0.36 & 0.36 \\
\hline $09-752$ & Black Holstein & AR, USA & 81.67 & 4.17 & 77.50 & 94.88 & 0.05 & 0.05 \\
\hline Arkansas Blackeye \#1 & Blackeye & AR, USA & 76.67 & 50.83 & 25.83 & 33.52 & 0.66 & 0.66 \\
\hline Early Acre & Cream & AR, USA & 67.50 & 32.50 & 35.00 & 50.92 & 0.49 & 0.49 \\
\hline Early scarlet & Pinkeye & AR, USA & 81.67 & 29.17 & 52.50 & 64.39 & 0.36 & 0.36 \\
\hline Ebony & Black & AR, USA & 90.00 & 69.17 & 20.83 & 23.06 & 0.77 & 0.77 \\
\hline ENCORE & Pinkeye & AR, USA & 71.67 & 30.00 & 41.67 & 58.10 & 0.42 & 0.42 \\
\hline Envoy & Red Holstein & AR, USA & 99.17 & 30.00 & 69.17 & 69.72 & 0.30 & 0.30 \\
\hline Epic Select & Tan & AR, USA & 81.67 & 10.83 & 70.83 & 86.92 & 0.13 & 0.13 \\
\hline EXCEL & Browneye & AR, USA & 86.67 & 70.00 & 16.67 & 19.24 & 0.81 & 0.81 \\
\hline PI152195 & Red & Paraguay & 82.50 & 44.17 & 38.33 & 46.71 & 0.53 & 0.54 \\
\hline PI152196 & Mixed (Cream Brown) & Paraguay & 95.00 & 58.33 & 36.67 & 39.42 & 0.61 & 0.62 \\
\hline PI152197 & Red & Paraguay & 88.33 & 25.00 & 63.33 & 71.42 & 0.29 & 0.28 \\
\hline PI152199 & Mixed (Purple Cream) & Paraguay & 79.17 & 45.83 & 33.33 & 42.02 & 0.58 & 0.58 \\
\hline PI162924 & Mixed (Cream Brown) & Paraguay & 83.33 & 66.67 & 16.67 & 18.72 & 0.81 & 0.79 \\
\hline PI167284 & Mixed (Cream Brown) & Turkey & 79.17 & 32.50 & 46.67 & 59.52 & 0.40 & 0.42 \\
\hline PI175332 & Tan & NA & 77.50 & 31.67 & 45.83 & 58.33 & 0.42 & 0.41 \\
\hline PI180014 & Tan & India & 71.67 & 27.50 & 44.17 & 62.76 & 0.37 & 0.40 \\
\hline PI190191 & Tan & Mexico & 63.33 & 17.50 & 45.83 & 72.09 & 0.28 & 0.28 \\
\hline PI201498 & Blackeye & Mexico & 92.50 & 77.50 & 15.00 & 16.24 & 0.84 & 0.84 \\
\hline PI218123 & Browneye & Pakistan & 97.50 & 30.83 & 66.67 & 68.22 & 0.32 & 0.32 \\
\hline PI221730 & Red Holstein & South_Africa & 69.17 & 30.83 & 38.33 & 55.17 & 0.45 & 0.44 \\
\hline PI221731 & Red Holstein & South_Africa & 95.83 & 50.83 & 45.00 & 46.87 & 0.53 & 0.53 \\
\hline PI223023 & Browneye & Iran & 95.00 & 56.67 & 38.33 & 40.74 & 0.59 & 0.60 \\
\hline PI225922 & Mixed (Brown Tan) & Zambia & 60.83 & 46.67 & 14.17 & 20.25 & 0.80 & 0.75 \\
\hline PI229551 & Browneye & Iran & 90.83 & 70.83 & 20.00 & 21.52 & 0.78 & 0.78 \\
\hline PI229734 & Blackeye & Iran & 88.33 & 26.67 & 61.67 & 70.13 & 0.30 & 0.31 \\
\hline PI244571 & Mixed (Brown Cream) & Guatemala & 66.67 & 17.50 & 49.17 & 73.74 & 0.26 & 0.26 \\
\hline PI250416 & Cream & Pakistan & 88.33 & 22.50 & 65.83 & 73.98 & 0.26 & 0.25 \\
\hline PI250587 & Dark brown & Madagascar & 70.00 & 5.83 & 64.17 & 91.73 & 0.08 & 0.08 \\
\hline PI253428 & Dark brown & Spain & 97.50 & 38.33 & 59.17 & 60.67 & 0.39 & 0.39 \\
\hline PI255765 & Blackeye & Nigeria & 97.50 & 56.67 & 40.83 & 41.85 & 0.58 & 0.58 \\
\hline PI255815 & Browneye & Nigeria & 77.50 & 54.17 & 23.33 & 28.81 & 0.71 & 0.69 \\
\hline PI257463 & Red Holstein & Nigeria & 79.17 & 46.67 & 32.50 & 40.51 & 0.59 & 0.59 \\
\hline PI262179 & Blackeye & Portugal & 73.33 & 55.00 & 18.33 & 24.68 & 0.75 & 0.75 \\
\hline PI292897 & Grey & Hungary & 67.50 & 28.33 & 39.17 & 58.30 & 0.42 & 0.42 \\
\hline PI292898 & Black & Hungary & 86.67 & 71.67 & 15.00 & 17.09 & 0.83 & 0.83 \\
\hline PI292899 & Tan & Hungary & 66.67 & 11.67 & 55.00 & 83.70 & 0.16 & 0.19 \\
\hline PI292913 & Tan & Hungary & 62.50 & 8.33 & 54.17 & 86.71 & 0.13 & 0.13 \\
\hline PI293467 & Mixed (Tan Brown) & USA & 84.17 & 28.33 & 55.83 & 66.45 & 0.34 & 0.34 \\
\hline PI293469 & Tan & NA & 91.67 & 23.33 & 68.33 & 74.72 & 0.25 & 0.26 \\
\hline PI293476 & Variable (Grey Brown) & USA & 70.83 & 7.50 & 63.33 & 87.68 & 0.12 & 0.09 \\
\hline PI293514 & Grey & USA & 78.33 & 21.67 & 56.67 & 72.51 & 0.27 & 0.28 \\
\hline PI293545 & Mixed (Brown Cream) & NA & 93.33 & 65.00 & 28.33 & 30.37 & 0.70 & 0.70 \\
\hline PI293570 & Mixed (Brown Tan) & NA & 62.50 & 7.50 & 55.00 & 87.63 & 0.12 & 0.12 \\
\hline PI293582 & Grey & NA & 70.00 & 22.50 & 47.50 & 68.13 & 0.32 & 0.33 \\
\hline PI293584 & Variable (Red Brown Cream) & NA & 67.50 & 58.33 & 9.17 & 13.31 & 0.87 & 0.86 \\
\hline PI297561 & Mixed (Brown Grey) & NA & 61.67 & 36.67 & 25.00 & 38.75 & 0.61 & 0.60 \\
\hline PI339587 & Red Holstein & South_Africa & 70.00 & 53.33 & 16.67 & 23.46 & 0.77 & 0.76 \\
\hline
\end{tabular}


Table 1. (Continued) Cowpea name (accession number), seed color, origin, and germination rates without salt treatment and under salt stress, absolute decrease (AD), inhibition index (II), relative salt tolerance (RST), and salt tolerance index (STI).

\begin{tabular}{|c|c|c|c|c|c|c|c|c|}
\hline Accession number & Seed color & Origin & Nonstress $(\%)$ & Stress (\%) & AD_( $\%)$ & II_(\%) & RST & STI \\
\hline$\overline{\text { PI339591 }}$ & Brown Holstein & South_Africa & 65.00 & 51.67 & 13.33 & 18.60 & 0.81 & 0.79 \\
\hline PI339592 & Brown & South_Africa & 70.83 & 44.17 & 26.67 & 36.71 & 0.63 & 0.62 \\
\hline PI339594 & Variable (Red Holstein Red Tan) & NA & 71.67 & 51.67 & 20.00 & 23.91 & 0.76 & 0.71 \\
\hline PI339609 & Tan & Tanzania & 89.17 & 50.00 & 39.17 & 43.40 & 0.57 & 0.56 \\
\hline PI339610 & Variable (Red Brown Cream) & Tanzania & 60.83 & 38.33 & 22.50 & 36.71 & 0.63 & 0.63 \\
\hline PI339611 & Tan & Tanzania & 73.33 & 62.50 & 10.83 & 14.60 & 0.85 & 0.85 \\
\hline PI339613 & Variable (Grey Tan Brown) & Tanzania & 93.33 & 45.00 & 48.33 & 51.62 & 0.48 & 0.48 \\
\hline PI347639 & Tan & NA & 60.00 & 36.67 & 23.33 & 38.34 & 0.62 & 0.61 \\
\hline PI353045 & Red Holstein & India & 77.50 & 45.83 & 31.67 & 40.68 & 0.59 & 0.59 \\
\hline PI353062 & Mixed (Cream Purple) & India & 94.17 & 68.33 & 25.83 & 27.51 & 0.72 & 0.73 \\
\hline PI367921 & Tan & NA & 89.17 & 73.33 & 15.83 & 17.68 & 0.82 & 0.82 \\
\hline PI406290 & Browneye & Mozambique & 65.00 & 18.33 & 46.67 & 72.02 & 0.28 & 0.28 \\
\hline PI430687 & Red & China & 68.33 & 32.50 & 35.83 & 51.75 & 0.48 & 0.47 \\
\hline PI487518 & Tan & Indonesia & 98.33 & 44.17 & 54.17 & 55.26 & 0.45 & 0.45 \\
\hline PI527561 & Tan & Burindi & 96.67 & 58.33 & 38.33 & 39.64 & 0.60 & 0.60 \\
\hline PI578902 & Red & China & 78.33 & 22.50 & 55.83 & 69.99 & 0.30 & 0.28 \\
\hline PI578907 & Black & China & 87.50 & 44.17 & 43.33 & 49.38 & 0.51 & 0.51 \\
\hline PI578911 & Tan & China & 82.50 & 47.50 & 35.00 & 41.91 & 0.58 & 0.57 \\
\hline PI582340 & Mixed (Brown Pink) & NA & 69.17 & 26.67 & 42.50 & 61.38 & 0.39 & 0.39 \\
\hline PI582347 & Tan & Myanmar & 80.83 & 49.17 & 31.67 & 39.10 & 0.61 & 0.61 \\
\hline PI582352 & Blackeye & Saudi_Arabia & 88.33 & 49.17 & 39.17 & 43.35 & 0.57 & 0.55 \\
\hline PI582354 & Blackeye & NA & 69.17 & 40.83 & 28.33 & 39.97 & 0.60 & 0.58 \\
\hline PI582415 & Tan & Mexico & 89.17 & 52.50 & 36.67 & 41.13 & 0.59 & 0.59 \\
\hline PI582416 & Variable (Red Brown Cream) & Mexico & 92.50 & 25.00 & 67.50 & 72.94 & 0.27 & 0.27 \\
\hline PI582420 & Variable (Red Brown) & NA & 77.50 & 65.00 & 12.50 & 15.36 & 0.85 & 0.83 \\
\hline PI582421 & Grey & NA & 97.50 & 65.00 & 32.50 & 33.50 & 0.66 & 0.67 \\
\hline PI582422 & Blackeye & NA & 75.83 & 70.00 & 5.83 & 7.67 & 0.92 & 0.92 \\
\hline PI582425 & Mixed (Tan Brown) & NA & 82.50 & 40.83 & 41.67 & 50.44 & 0.50 & 0.49 \\
\hline PI582428 & Blackeye & Trinidad and Tobago & 64.72 & 49.17 & 15.56 & 22.14 & 0.78 & 0.76 \\
\hline PI582467 & Black Holstein & NA & 75.83 & 37.50 & 38.33 & 49.07 & 0.51 & 0.49 \\
\hline PI582474 & Purple & Botswana & 64.17 & 25.83 & 38.33 & 59.41 & 0.41 & 0.40 \\
\hline PI582522 & Tan & Mexico & 95.00 & 0.83 & 94.17 & 99.12 & 0.01 & 0.01 \\
\hline PI582531 & Variable (Grey Brown Tan) & Ghana & 65.83 & 42.50 & 23.33 & 31.66 & 0.68 & 0.62 \\
\hline PI582542 & Browneye & Mexico & 88.33 & 58.33 & 30.00 & 33.67 & 0.66 & 0.66 \\
\hline PI582551 & Blackeye & Botswana & 84.17 & 64.17 & 20.00 & 23.85 & 0.76 & 0.76 \\
\hline PI582570 & Browneye & India & 67.78 & 58.33 & 9.44 & 13.65 & 0.86 & 0.86 \\
\hline PI582573 & Browneye & Kenya & 67.50 & 53.33 & 14.17 & 20.60 & 0.79 & 0.79 \\
\hline PI582574 & Tan & Kenya & 75.00 & 28.33 & 46.67 & 61.01 & 0.39 & 0.38 \\
\hline PI582575 & Black & Kenya & 91.67 & 28.33 & 63.33 & 68.91 & 0.31 & 0.31 \\
\hline PI582578 & Tan & Kenya & 77.50 & 42.50 & 35.00 & 41.86 & 0.58 & 0.53 \\
\hline PI582579 & Tan & Kenya & 97.50 & 21.67 & 75.83 & 77.66 & 0.22 & 0.22 \\
\hline PI582665 & Variable (Grey Brown Tan) & Botswana & 89.17 & 1.67 & 87.50 & 98.20 & 0.02 & 0.02 \\
\hline PI582666 & Tan & Botswana & 83.33 & 46.67 & 36.67 & 44.14 & 0.56 & 0.56 \\
\hline PI582680 & Browneye & Botswana & 65.83 & 26.67 & 39.17 & 59.28 & 0.41 & 0.41 \\
\hline PI582696 & Brown & Botswana & 74.17 & 44.17 & 30.00 & 40.27 & 0.60 & 0.59 \\
\hline PI582703 & Red Holstein & Botswana & 69.17 & 27.50 & 41.67 & 60.29 & 0.40 & 0.40 \\
\hline PI582789 & Tan & Kenya & 95.83 & 36.67 & 59.17 & 61.62 & 0.38 & 0.38 \\
\hline PI582809 & Tan & Botswana & 61.67 & 23.33 & 38.33 & 62.28 & 0.38 & 0.38 \\
\hline PI582813 & Mixed(Grey Cream Black eye) & Botswana & 77.50 & 0.00 & 77.50 & 100.00 & 0.00 & 0.00 \\
\hline PI582815 & Purple & Botswana & 84.17 & 41.67 & 42.50 & 50.45 & 0.50 & 0.49 \\
\hline PI582818 & Black Holstein & Botswana & 90.00 & 20.00 & 70.00 & 77.74 & 0.22 & 0.22 \\
\hline PI582821 & Mixed (Brown Tan) & Botswana & 90.83 & 40.00 & 50.83 & 56.15 & 0.44 & 0.44 \\
\hline PI582852 & Brown & Botswana & 61.67 & 31.67 & 30.00 & 48.31 & 0.52 & 0.51 \\
\hline PI582863 & Browneye & Botswana & 87.50 & 59.17 & 28.33 & 32.17 & 0.68 & 0.68 \\
\hline PI582873 & Tan & Botswana & 67.50 & 7.50 & 60.00 & 88.53 & 0.11 & 0.11 \\
\hline PI582874 & Tan & Botswana & 85.00 & 37.50 & 47.50 & 55.91 & 0.44 & 0.45 \\
\hline PI582875 & Tan & Botswana & 70.83 & 11.67 & 59.17 & 83.54 & 0.16 & 0.17 \\
\hline PI582878 & Green & NA & 72.50 & 12.50 & 60.00 & 81.76 & 0.18 & 0.16 \\
\hline PI582894 & Tan & Botswana & 71.67 & 27.50 & 44.17 & 61.35 & 0.39 & 0.38 \\
\hline PI582932 & Browneye & Malawi & 70.00 & 57.50 & 12.50 & 17.56 & 0.82 & 0.82 \\
\hline PI583193 & Variable (Gray Brown) & Senegal & 94.17 & 15.83 & 78.33 & 83.11 & 0.17 & 0.17 \\
\hline PI583194 & Variable (Tan Grey) & Senegal & 99.17 & 9.17 & 90.00 & 90.83 & 0.09 & 0.09 \\
\hline PI583198 & Tan & Senegal & 96.67 & 57.50 & 39.17 & 40.82 & 0.59 & 0.60 \\
\hline PI583201 & Browneye & Senegal & 70.83 & 51.67 & 19.17 & 24.75 & 0.75 & 0.73 \\
\hline PI583202 & Browneye & Senegal & 89.17 & 56.67 & 32.50 & 36.56 & 0.63 & 0.64 \\
\hline PI583209 & Tan & Nigeria & 90.83 & 23.33 & 67.50 & 74.27 & 0.26 & 0.26 \\
\hline PI583244 & Tan & Senegal & 80.83 & 35.83 & 45.00 & 55.53 & 0.44 & 0.44 \\
\hline PI583248 & Browneye & Senegal & 77.78 & 63.33 & 14.44 & 18.13 & 0.82 & 0.81 \\
\hline PI583249 & Browneye & Senegal & 69.17 & 47.50 & 21.67 & 30.62 & 0.69 & 0.69 \\
\hline PI583274 & Browneye & Senegal & 88.33 & 62.50 & 25.83 & 28.92 & 0.71 & 0.71 \\
\hline PI583487 & Tan & Senegal & 62.50 & 39.17 & 23.33 & 33.91 & 0.66 & 0.61 \\
\hline PI583488 & Brown & Australia & 69.17 & 30.83 & 38.33 & 55.67 & 0.44 & 0.45 \\
\hline PI583550 & Purple & Mali & 93.33 & 42.50 & 50.83 & 54.27 & 0.46 & 0.45 \\
\hline
\end{tabular}


Table 1. (Continued) Cowpea name (accession number), seed color, origin, and germination rates without salt treatment and under salt stress, absolute decrease (AD), inhibition index (II), relative salt tolerance (RST), and salt tolerance index (STI).

\begin{tabular}{|c|c|c|c|c|c|c|c|c|}
\hline Accession number & Seed color & Origin & Nonstress (\%) & Stress (\%) & $\overline{A D \_(\%)}$ & II_(\%) & RST & $\overline{\text { STI }}$ \\
\hline$\overline{\text { PI583551 }}$ & Browneye & Mali & 70.83 & 46.67 & 24.17 & 33.91 & 0.66 & 0.67 \\
\hline PI610520 & Black & Italy & 69.17 & 12.50 & 56.67 & 81.33 & 0.19 & 0.18 \\
\hline PI610533 & Blackeye & $\mathrm{NA}$ & 80.00 & 49.17 & 30.83 & 37.96 & 0.62 & 0.61 \\
\hline PI610604 & Purple & Italy & 65.83 & 23.33 & 42.50 & 64.19 & 0.36 & 0.37 \\
\hline PI666252 & Tan & USA & 90.00 & 26.67 & 63.33 & 70.33 & 0.30 & 0.30 \\
\hline
\end{tabular}
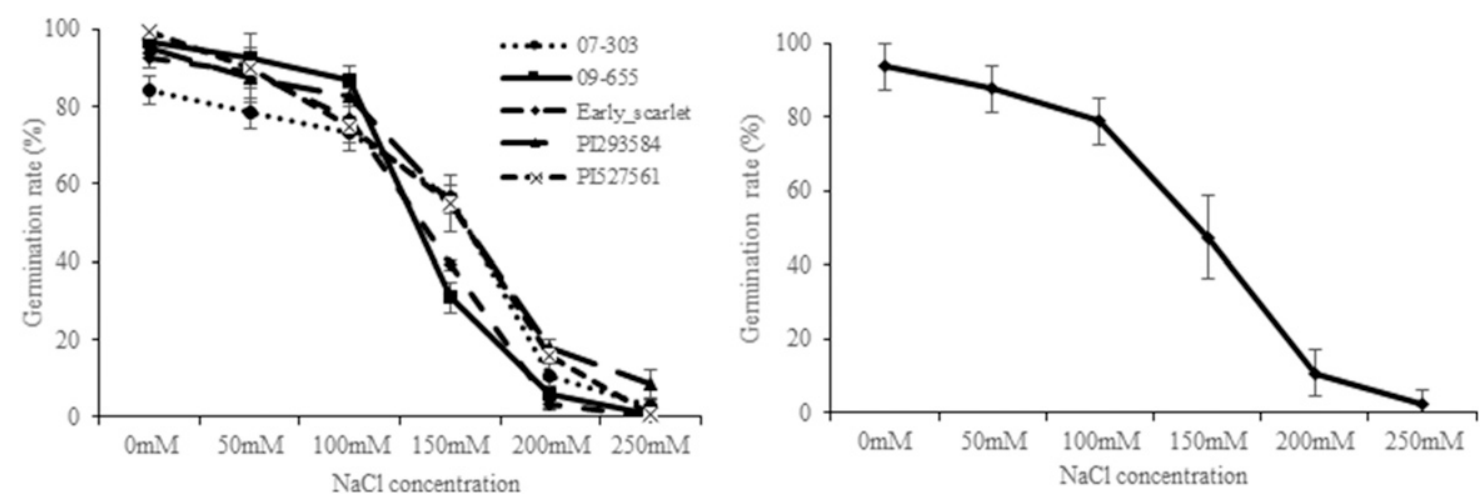

Fig. 1. Seed germination rates under six salt $(\mathrm{NaCl})$ concentrations $(0,50,100,150,200$, and $250 \mathrm{~mm})$ : (A) five cowpea genotypes, respectively, and $(\mathbf{B})$ the pooled five cowpea genotypes.

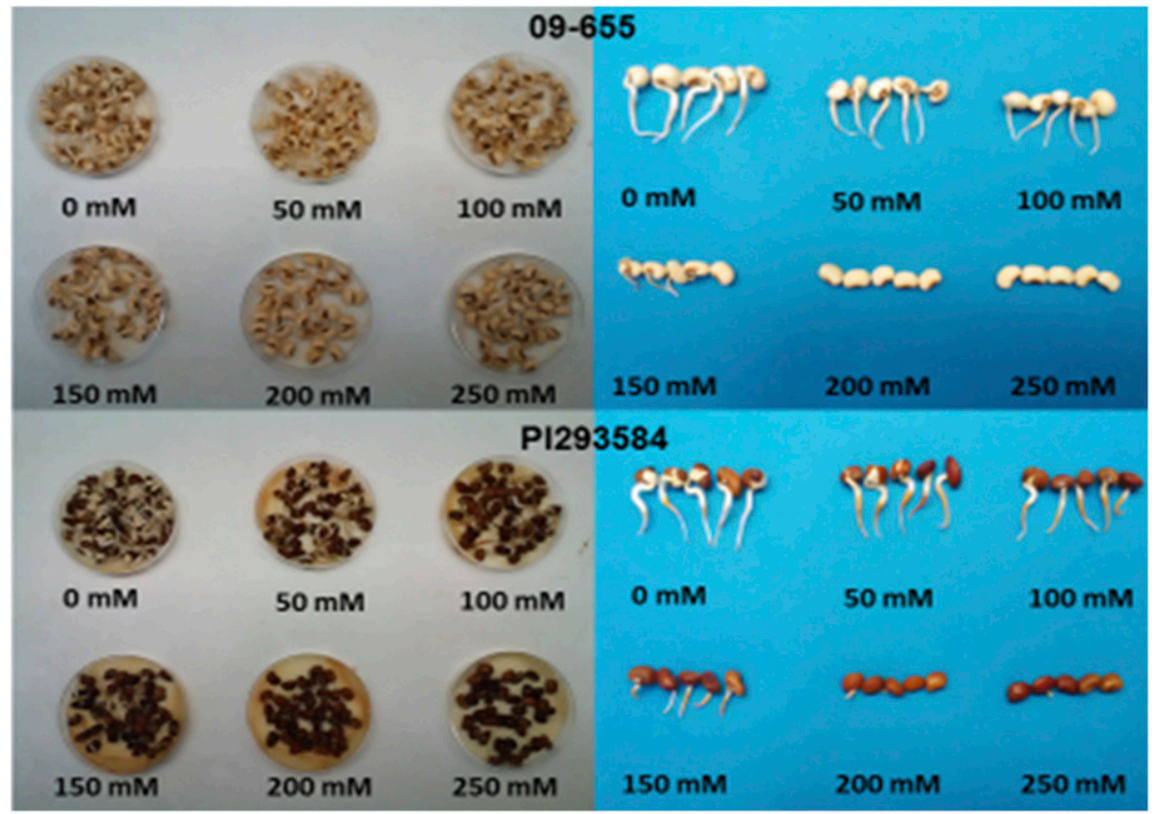

Fig. 2. An example of the seed germination rate in two cowpea genotypes, '09-655' and 'PI293584' under six salt $(\mathrm{NaCl})$ concentrations in the petri dishes (left) and the radicle length (right) in $48 \mathrm{~h}$ after $\mathrm{NaCl}$ treatment at $28^{\circ} \mathrm{C}$ condition.

$\sigma_{\mathrm{G}}^{2}, \sigma_{\mathrm{E}}^{2}$, and $\sigma_{\mathrm{S}}^{2}$ were obtained using the following formulas:

$$
\begin{aligned}
& \sigma_{\mathrm{G}}^{2}=(\mathrm{MSG}-\mathrm{MSE}) / \mathrm{r}, \sigma_{\mathrm{E}}^{2}=\mathrm{MSE}, \\
& \sigma_{\mathrm{S}}^{2}=(\mathrm{MSS}-\mathrm{MSE}) / \mathrm{n}
\end{aligned}
$$

where $\mathrm{MSG}=$ mean square genotype, $\mathrm{MSE}=$ mean square error, MSS = mean square shelve, $r=$ number of replications, and $n=$ number of genotypes.

In addition, ANOVA of the effects of the origin (region) of the cowpea genotypes on salt tolerance were analyzed using GLM of JMP Genomics. Before analysis, the genotypes without any information on their origin were discarded so that 132 genotypes were analyzed in this study.

The cluster analysis involved 151 individuals which were measured using six parameters: germination rates without salt treatment (nonstress) and under salt stress (stress), AD, II, RST, and STI. Ward's method was used as a clustering technique (Sahu, 2013). The phylogenetic tree diagram was constructed using "Multivariate Methods" by "Cluster" in JMP Genomics 7 (SAS Institute, Cary, $\mathrm{NC})$.

\section{Results and Discussion}

Optimal concentration for evaluation of cowpea salt tolerance at germination stage. From our preliminary experiments with six salt concentrations: $0,50,100,150,200$, and $250 \mathrm{~mm}$ of $\mathrm{NaCl}$, when the $\mathrm{NaCl}$ concentration increased, the seed germination rate decreased either pooled across cowpea genotypes or individually (Figs. 1 and 2; Tables 2 and 3). At the salinity level at $250 \mathrm{~mm}$, the seeds had minimal germination rate which made it difficult to separate the tolerant from the susceptible genotypes (Tables 2 and 3 ). At $150 \mathrm{~mm}$, seed germination differed the most among the cowpea genotypes ( $F=$ $27.37, P$ value $=0.0001)($ Table 3$)$. It was reported that increasing salt concentration reduced the germination rate of cowpea genotype in other studies (Thiam et al., 2013; Wests and Francois, 1982; Zahedi et al., 2012). Lobato et al. (2009) used $150 \mathrm{~mm} \mathrm{NaCl}$ to conduct their study on the effects of salinity on cowpea germination. Therefore, the salt concentration of $150 \mathrm{~mm} \mathrm{NaCl}$ would be a reasonable concentration to perform salt tolerance testing in cowpea and it was used in our study.

Germination at nonsalt stress and salt stress conditions. The average germination rate among the 151 cowpea genotypes under the nonsalt stress condition varied from $60.0 \%$ to $99.2 \%$, with a mean of $80.2 \%$ and a standard deviation of $11.0 \%$. Incubating seeds in $150 \mathrm{~mm} \mathrm{NaCl}$ resulted in a drop of 
germination to $0 \%$ to $77.5 \%$, with an overall average of $38.7 \%$ and a standard deviation of $18.3 \%$. These results suggested that salinity significantly reduced the germination rate in cowpea. For all parameters, there were no significant incubator shelf effects. The germination rate was significantly different among the cowpea genotypes under nonstress condition $(F=7.37, P$ value $<0.0001)$ (Table 4). 'Envoy' (99.2\%), 'PI583194' (99.2\%), 'PI487518' (98.3\%), 'PI582579' (97.5\%), 'PI218123' (97.5\%), 'PI253428' (97.5\%), 'PI255765' (97.5\%), and 'PI582421' $(97.5 \%)$ had the highest germination rates, and 'PI225922' (60.8\%), 'PI339610' (60.8\%), and 'PI347639' (60\%) had the lowest germination rates. Under salinity condition, 'PI201498' performed well with a germination rate of $77.5 \%$, indicating it was a salt-tolerant cowpea accession at germination stage; however, the lowest germination rate was recorded for 'PI252665' (1.7\%), '09-393' (0.8\%), 'PI582522' (0.8\%), and 'PI582813' (0.0\%), indicating that they were very susceptible to salt stress at the germination stage. The germination rate among the cowpea genotypes was significantly different under salt stress $(F=16.62, P$ value $<0.0001)$ (Table 4).

Absolute decrease and inhibition index. The AD indicated the decrease of the germination rate between the nonsalinity conditions and the salt treatment. In this study, salinity reduced the germination rate from $5.8 \%$ to $94.2 \%$. The cowpea genotypes responded differently to salinity environment at germination stage in terms of $\mathrm{AD}(F=$ 10.1, $P$ value $<0.0001$ ) (Table 4). 'PI582522'
(94.2\%) exhibited the highest AD. 'PI585422' had the lowest $\mathrm{AD}, 5.8 \%$. The higher the $\mathrm{AD}$ was, the more salt susceptible the genotype.

The II was a parameter which was widely used for studies related to plant stress (González, 1996). The II of the germination ranged from $7.7 \%$ to $100.0 \%$, with an average of $51.2 \%$ and a standard deviation of $22.6 \%$, indicating a large variability in responses to salinity among the cowpea genotypes. In addition, the inhibition of the germination because of salinity significantly differed among the cowpea genotypes $(F=11.6, P$ value $<0.0001$ ) (Table 4). 'PI582813', 'PI582522', and '09-393' had a very high II, over $99 \%$ under salt stress. These results suggested that these lines are highly salt sensitive at germination stage. 'PI582422', '09-529', 'PI293584', 'PI582570', and

Table 2. ANOVA for pooled seed germination rates of five cowpea genotypes under six NaCl concentrations (0, 50, 100, 150, 200, and 250 mu).

\begin{tabular}{|c|c|c|c|c|c|}
\hline Source & $\mathrm{DF}$ & Sum of squares & Mean square & F ratio & Prob $>F$ \\
\hline Shelf & 2 & 2.534 & 1.267 & 0.5061 & 0.6175 \\
\hline Concentration & 5 & $23,605.7$ & $4,721.1472$ & $1,885.53$ & $<0.0001$ \\
\hline Error & 10 & 25.039 & 2.5 & & \\
\hline
\end{tabular}

$\overline{\mathrm{DF}}=$ degree(s) of freedom.

Table 3. ANOVA for seed germination rates of five cowpea genotypes under six salt concentrations of NaCl.

\begin{tabular}{|c|c|c|c|c|c|c|}
\hline $\mathrm{NaCl}$ concn $(\mathrm{mM})$ & Source & $\mathrm{DF}$ & Sum of squares & Mean square & $\mathrm{F}$ ratio & Prob $>F$ \\
\hline \multirow[t]{3}{*}{$\overline{0}$} & Shelf & 2 & 7.50 & 3.75 & 0.2553 & 0.7807 \\
\hline & Genotype & 4 & 397.50 & 99.38 & 6.766 & 0.0111 \\
\hline & Error & 8 & 117.50 & 14.69 & & \\
\hline \multirow[t]{3}{*}{50} & Shelf & 2 & 22.50 & 11.25 & 0.4269 & 0.6666 \\
\hline & Genotype & 4 & 354.17 & 88.54 & 3.3597 & 0.068 \\
\hline & Error & 8 & 210.83 & 26.35 & & \\
\hline \multirow[t]{3}{*}{100} & Shelf & 2 & 40.83 & 20.42 & 1.0262 & 0.4011 \\
\hline & Genotype & 4 & 373.33 & 93.33 & 4.6911 & 0.0304 \\
\hline & Error & 8 & 159.17 & 19.90 & & \\
\hline \multirow[t]{3}{*}{150} & Shelf & 2 & 55.83 & 27.92 & 1.8741 & 0.215 \\
\hline & Genotype & 4 & $1,630.83$ & 407.71 & 27.3706 & 0.0001 \\
\hline & Error & 8 & 119.17 & 14.90 & & \\
\hline \multirow[t]{3}{*}{200} & Shelf & 2 & 3.70 & 1.85 & 0.2441 & 0.789 \\
\hline & Genotype & 4 & 458.57 & 114.64 & 15.1259 & 0.0008 \\
\hline & Error & 8 & 60.63 & 7.58 & & \\
\hline \multirow[t]{3}{*}{250} & Shelf & 2 & 7.50 & 3.75 & 0.7059 & 0.522 \\
\hline & Genotype & 4 & 137.50 & 34.38 & 6.4706 & 0.0126 \\
\hline & Error & 8 & 42.50 & 5.31 & & \\
\hline
\end{tabular}

$\mathrm{DF}=$ degree(s) of freedom.

Table 4. ANOVA for six parameters related to seed germination based on cowpea genotypes.

\begin{tabular}{|c|c|c|c|c|c|c|c|}
\hline Parameters $^{\mathrm{z}}$ & Source & DF & Sum of squares & Mean square & $\mathrm{F}$ ratio & Prob $>$ F & Estimated Mean square \\
\hline \multirow[t]{3}{*}{ Germination without salt stress (nonstress) } & Shelf & 2 & 214.528 & 107.264 & 2.163 & 0.1167 & $\sigma_{\mathrm{e}}^{2}+\mathrm{n}^{*} \sigma_{\mathrm{s}}^{2}$ \\
\hline & Genotype & 150 & $54,845.211$ & 365.635 & 7.374 & $<0.0001$ & $\sigma_{\mathrm{e}}^{2}+\mathrm{r}^{*} \sigma_{\mathrm{g}}^{2}$ \\
\hline & Error & 300 & $14,875.217$ & 49.584 & & & $\sigma_{\mathrm{e}}^{2}$ \\
\hline \multirow[t]{3}{*}{ Germination under $150 \mathrm{~mm} \mathrm{NaCl}$ (stress) } & Shelf & 2 & 86.865 & 43.433 & 0.715 & 0.4899 & $\sigma_{\mathrm{e}}^{2}+\mathrm{n}^{*} \sigma_{\mathrm{s}}^{2}$ \\
\hline & Genotype & 150 & $151,389.901$ & $1,009.266$ & 16.621 & $<0.0001$ & $\sigma_{\mathrm{e}}^{2}+\mathrm{r}^{*} \sigma_{\mathrm{g}}^{2}$ \\
\hline & Error & 300 & $18,217.301$ & 60.724 & & & $\sigma_{\mathrm{e}}^{2}$ \\
\hline \multirow[t]{3}{*}{ Absolute decrease (AD) } & Shelf & 2 & 55.025 & 27.513 & 0.245 & 0.7828 & $\sigma_{\mathrm{e}}^{2}+\mathrm{n}^{*} \sigma_{\mathrm{s}}^{2}$ \\
\hline & Genotype & 150 & $169,967.007$ & $1,133.113$ & 10.093 & $<0.0001$ & $\sigma_{\mathrm{e}}^{2}+\mathrm{r}^{*} \sigma_{\mathrm{g}}^{2}$ \\
\hline & Error & 300 & $33,681.420$ & 112.271 & & & $\sigma_{\mathrm{e}}^{2}$ \\
\hline \multirow[t]{3}{*}{ Inhibition index (II) } & Shelf & 2 & 40.441 & 20.221 & 0.153 & 0.8581 & $\sigma_{\mathrm{e}}^{2}+\mathrm{n}^{*} \sigma_{\mathrm{s}}^{2}$ \\
\hline & Genotype & 150 & $229,783.382$ & $1,531.889$ & 11.597 & $<0.0001$ & $\sigma_{\mathrm{e}}^{2}+\mathrm{r}^{*} \sigma_{\mathrm{g}}^{2}$ \\
\hline & Error & 300 & $39,629.810$ & 132.099 & & & $\sigma_{\mathrm{e}}^{2}$ \\
\hline \multirow[t]{3}{*}{ Relative salt tolerance (RST) } & Shelf & 2 & 0.004 & 0.002 & 0.139 & 0.8699 & $\sigma_{\mathrm{e}}^{2}+\mathrm{n}^{*} \sigma_{\mathrm{s}}^{2}$ \\
\hline & Genotype & 150 & 22.947 & 0.153 & 11.587 & $<0.0001$ & $\sigma_{\mathrm{e}}^{2}+\mathrm{r}^{*} \sigma_{\mathrm{g}}^{2}$ \\
\hline & Error & 300 & 3.961 & 0.013 & & & $\sigma_{\mathrm{e}}^{2}$ \\
\hline \multirow[t]{3}{*}{ Salt tolerance index (STI) } & Shelf & 2 & 0.058 & 0.029 & 2.560 & 0.079 & $\sigma_{\mathrm{e}}^{2}+\mathrm{n}^{*} \sigma_{\mathrm{s}}^{2}$ \\
\hline & Genotype & 150 & 22.465 & 0.150 & 13.164 & $<0.0001$ & $\sigma_{\mathrm{e}}^{2}+\mathrm{r}^{*} \sigma_{\mathrm{g}}^{2}$ \\
\hline & Error & 300 & 3.413 & 0.011 & & & $\sigma_{\mathrm{e}}^{2}$ \\
\hline
\end{tabular}

${ }^{\mathrm{z}}$ Germination rate under non-salinity condition, salt stress, and different formulas used to assess genotype performed to salinity.

$\mathrm{DF}=$ degree(s) of freedom. 
'PI339611' had the lowest II, which were $7.7 \%, 12.2 \%, 13.3 \%, 13.6 \%$, and $14.6 \%$, respectively. These accessions could be excellent sources for salt tolerance at germination stage. The lower the II was, the more likely the genotype withstood salt stress (González, 1996).

Relative salt tolerance and salt-tolerance index. The RST and STI were two parameters to measure cowpea salt tolerance. The higher the RST or STI parameter was, the more likely the genotype was salt tolerant (Fernandez, 1992; Saad et al., 2014).

The RST varied from 0 to 0.92 , with a mean of 0.49 and a standard deviation of 0.23 . RST was significantly different among the cowpea genotypes $(F=11.99, P$ value $<$ 0.0001) (Table 4). 'PI582422', '09-529', 'PI293584', and 'PI582570' had the highest RST scores, indicating that they were salt tolerant based on RST. 'PI582813', '09-393', 'PI582522', and 'PI582665' exhibited the lowest RST scores, suggesting that they were salt-sensitive at germination stage.

The STI ranged from 0 to 0.92 , with a mean of 0.48 and a standard deviation of
0.22 . Significant differences were observed among the cowpea accessions in terms of STI $(F=13.16, P$ value $<0.0001)$ (Table 4$)$. 'PI582422' (0.92), '09-529' (0.87), 'PI293584' (0.86), and 'PI582570' (0.85) exhibited the highest STI, suggesting that these lines were highly tolerant to salinity at germination stage. The lowest STI was found in 'PI582813' (0), '09-393' (0.01), 'PI582522' (0.01), and 'PI582665' (0.02) (Table 4), indicating that these cowpea genotypes are highly saltsensitive.

The population dynamic of a crop is closely related to its seed germination (TeKrony and Egli, 1991). In this study, large variation of responses to salinity among the cowpea genotypes was observed for all parameters. As expected, there was a significant effect of salinity on the germination rate of the cowpea panel. Similar results have been found in other studies (Ashebir et al., 2013; Taffouo et al., 2009; Wests and Francois, 1982).

Analysis by geographical location. Significant differences were observed in seed germination rate without salt treatment and the germination rate under salt stress, $\mathrm{AD}, \mathrm{II}$, RST, and STI among the 12 different regions of origin of the cowpea genotypes ( $P$ values $<$ 0.0001 ) (Table 5). Genotypes from the Caribbean and Southern Asia showed better salt tolerance at the germination stage. The II was smaller than $40 \%$, and both RST and STIs were greater than 0.6 on average. Cowpea genotypes from Europe and North America were more sensitive to salinity at the germination stage (Table 6). The results suggested that the origins of those genotypes markedly impacted their response to salinity, so the origin should be taken into a consideration when one selects cowpea genotypes for salt tolerance. Little has been done regarding the effect of geographical distribution on plant salt tolerance. However, geographical distribution proved to be a strong driving factor on plant adaptation to stress. Burke (1990) reported that crops grown in semiarid areas developed mechanisms which enabled them to overcome permanent exposure to high temperature. Those mechanisms involved both cellular adaptation and photosynthetic responses to heat stress, indicating

Table 5. ANOVA for six parameters related to seed germination based on the origin of cowpea genotypes.

\begin{tabular}{|c|c|c|c|c|c|c|}
\hline Parameter $^{2}$ & Source & $\mathrm{DF}$ & Sum of squares & Mean square & $\mathrm{F}$ ratio & Prob $>F$ \\
\hline Germination without salt stress (nonstress) & C. Total & 395 & $59,483.693$ & & & \\
\hline \multirow[t]{2}{*}{ Germination under $150 \mathrm{~mm} \mathrm{NaCl}$ (stress) } & Region & 11 & $9,729.556$ & 884.505 & 2.4725 & 0.0053 \\
\hline & C. Total & 395 & $147,101.073$ & & & \\
\hline \multirow[t]{2}{*}{ Absolute decrease (AD) } & Region & 11 & $10,023.452$ & 911.223 & 2.0521 & 0.0229 \\
\hline & Error & 384 & $170,513.885$ & 444.047 & & \\
\hline & Error & 384 & $217,873.973$ & 567.38 & & \\
\hline & C. Total & 395 & $232,649.145$ & & & \\
\hline \multirow[t]{3}{*}{ Relative salt tolerance(RST) } & Region & 11 & 1.479 & 0.134 & 2.3737 & 0.0076 \\
\hline & Error & 384 & 21.754 & 0.057 & & \\
\hline & C. Total & 395 & 23.233 & & & \\
\hline \multirow[t]{2}{*}{ Salt tolerance index (STI) } & Region & 11 & 1.411 & 0.128 & 2.3359 & 0.0086 \\
\hline & Error & 384 & 21.081 & 0.055 & & \\
\hline
\end{tabular}

${ }^{\mathrm{z}}$ Parameters indicate the germination rate under nonsalinity condition, salt stress, and different formulas used to assess genotype performed to salinity.

${ }^{\mathrm{y}}$ Geographical areas where cowpea genotypes originated.

$\mathrm{DF}=$ degree $(\mathrm{s})$ of freedom .

Table 6. Mean separation for salt tolerance parameters of different regions.

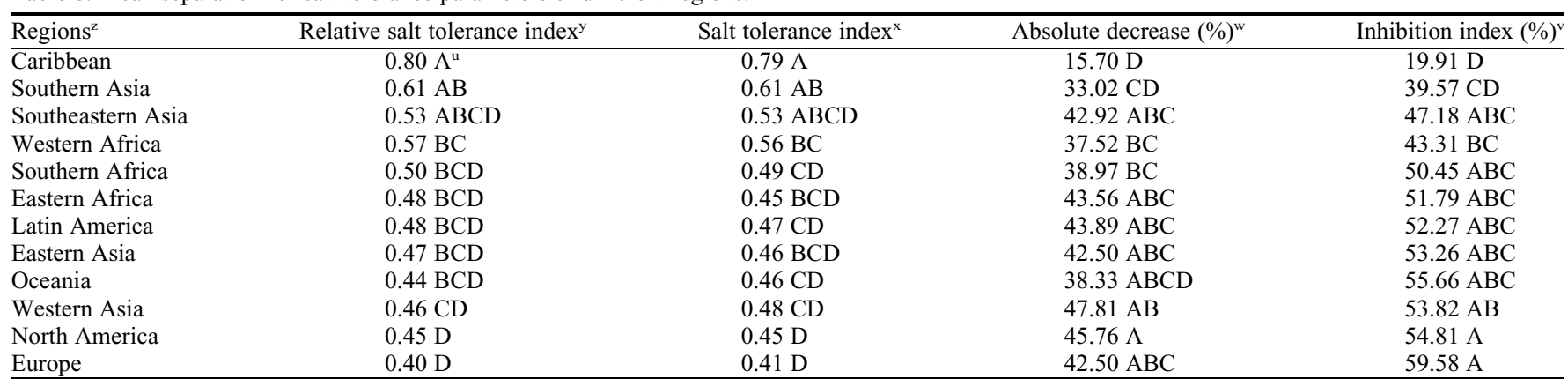

${ }^{\mathrm{z}}$ Geographical areas where cowpea genotypes originated.

${ }^{\mathrm{y}}$ Germination rate under salt stress condition divided by germination rate under nonsalt condition.

${ }^{\mathrm{x}}$ Germination rate under salt stress condition times germination rate under nonsalt condition divided by square of germination rate under nonsalt condition.

${ }^{\mathrm{w}}$ Germination rated under nonsalt condition subtracted by the germination under salt stress.

${ }^{\mathrm{v}}$ Germination rated under nonsalt condition subtracted by the germination under salt stress, divided by germination rate under nonsalt condition, and multiplied by one hundred.

${ }^{\mathrm{u}}$ Means followed by the same letter are not significantly different using the Student's $t$ test at alpha $=0.05$. 

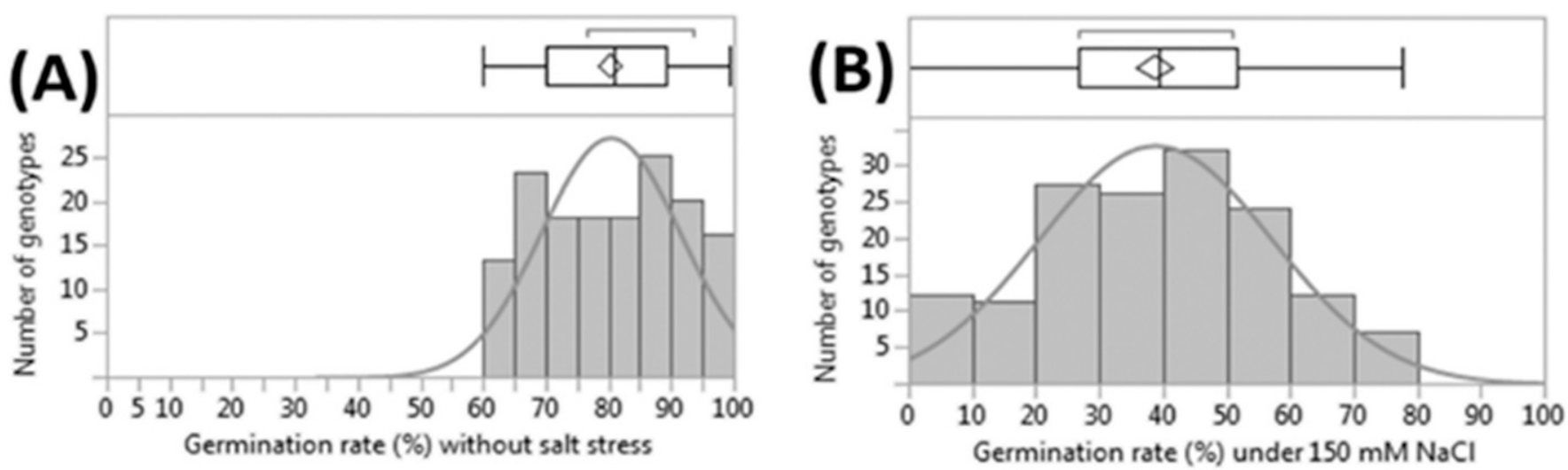

Fig. 3. Distribution of seed germination rates among 151 cowpea genotypes: (A) without salt stress and (B) with salt stress.
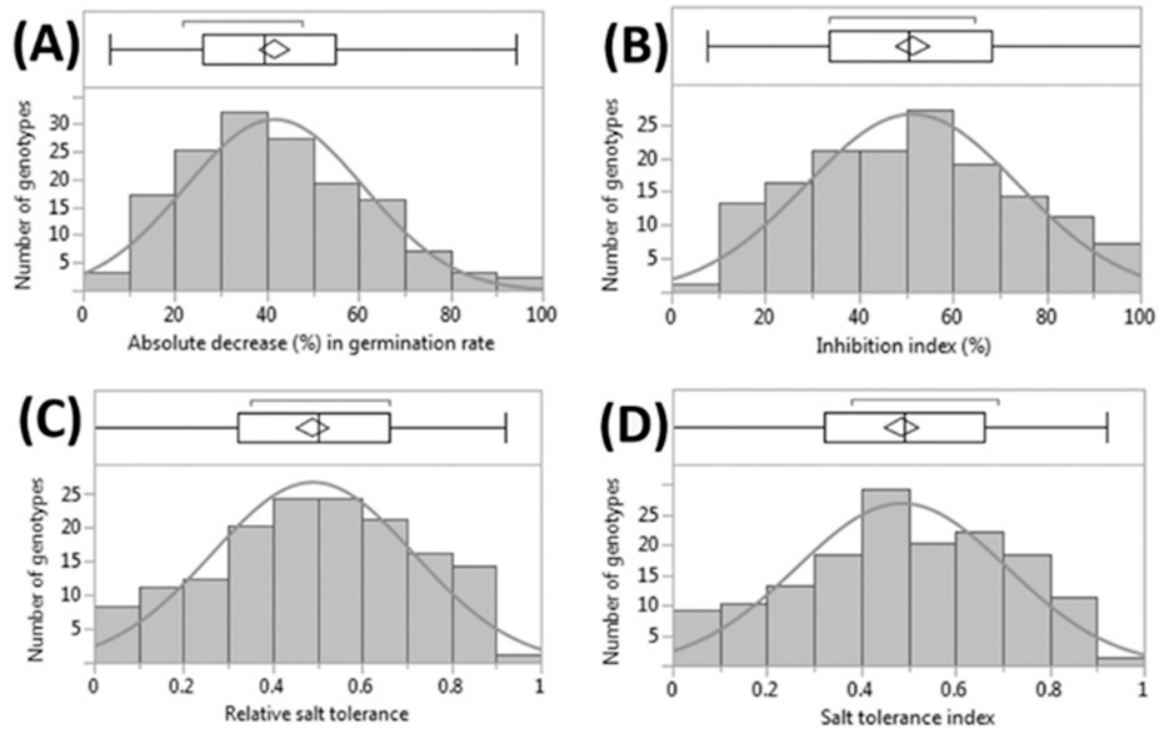

Fig. 4. Distributions of seed germination rates among 151 cowpea genotypes in four parameters: (A) absolute decrease, $(\mathbf{B})$ inhibition index, (C) relative salt tolerance, and (D) salt tolerance index.

Table 7. Correlation among six parameters, without salt stress (nonstress), with salt stress (stress), absolute decrease (AD), inhibition index (II), relative salt tolerance index (RST), and salt tolerance index (STI).

\begin{tabular}{lccccrr}
\hline Correlation & Nonstress & Stress & $\mathrm{AD}^{\mathrm{z}}$ & $\mathrm{II}^{\mathrm{y}}$ & $\mathrm{RST}^{\mathrm{x}}$ & $\mathrm{STI}^{\mathrm{w}}$ \\
\hline Nonstress & 1 & 0.20 & 0.38 & 0.10 & -0.10 & -0.08 \\
Stress & 0.20 & 1 & -0.83 & -0.95 & 0.95 & 0.95 \\
AD & 0.38 & -0.83 & 1 & 0.95 & -0.95 & -0.95 \\
II & 0.10 & -0.95 & 0.95 & 1 & -1 & -1 \\
RST & -0.10 & 0.95 & -0.95 & -1 & 1 & 1 \\
STI & -0.08 & 0.95 & -0.95 & -1 & 1 & 1 \\
\hline
\end{tabular}

${ }^{\mathrm{z}}$ Absolute decrease.

ynhibition index.

${ }^{\mathrm{x}}$ Relative salt tolerance.

${ }^{\mathrm{w}}$ Salt tolerance index.

'PI582813' and '09-393' were salt tolerant. In addition, Stankowski et al. (2015) showed that ecology played a pivotal role in plant adaptation. Their study on Mimulus aurantiacus C., a flower plant, revealed that floral trait had evolved according to the local conditions, suggesting that geographical location had shaped the adaptation of some traits to a specific environment. With respect to salt tolerance in cowpea, further investigation is required to unravel the mechanisms behind these significant differences in response to salinity among the genotypes from different countries.

Broad sense heritability. $\mathrm{H}^{2}$ associated with the seed germination without salt stress was $68 \%$. However, it was $83.9 \%$ under saline conditions. $\mathrm{H}^{2}$ was also observed as $75.2 \%$ for the $\mathrm{AD}$ in germination rate due to salt stress. The results indicated a high $\mathrm{H}^{2}$ for the parameters related to the II, RST, and STI, with $\mathrm{H}^{2}$ equal to $77.9 \%, 78.2 \%$, and
$80.8 \%$, respectively. Foolad and Jones (1992) found high heritability for tomato salt tolerance at seed germination stage. They estimated heritability to be $76.0 \%$ for tomato salt tolerance of an $\mathrm{F}_{2: 3}$ population derived from a cross between 'PI174263' and 'UCT5', which was close to those of salt tolerance in cowpea in this current investigation.

Correlation between parameters. The six parameters, without salt stress (nonstress), with salt stress (stress), AD, RST, and STI involved in this study showed near normal distributions (Figs. 3 and 4). The correlation coefficients among the six parameters were estimated (Table 7). There was a relatively low linear correlation between the germination rate under nonsalt stress condition and the other five parameters related to salinity stress, with $r=0.20,0.38,0.10,-0.10$, and 0.08 , respectively (Table 7 ), suggesting that salt tolerance at germination stage had a weak association with the germination rate in normal conditions in cowpea. However, the seed germination rate under salt stress had a very high negative linear correlation with the AD $(r=-0.83)$ and the II $(r=-0.95)$, but a high positive linear correlation observed between the germination rate under salt treatment and the RST $(r=0.95)$, and the STI $(r=0.95)$. These results indicate that salt tolerance at germination stage is highly associated with germination rate under salt stress.

Cluster analysis. Five different groups were identified among the 151 cowpea genotypes involved in this study (Supplemental Fig. 1). The three cowpea accessions, 'PI582422', 'PI293584', and 'PI582570' having the highest STI were clustered together and located at the same cluster, and the four lowest STI genotypes, 'PI582813', '09-393', 'PI582522', and 'PI582665' were clustered together and belonged to another cluster. These results indicated that the STI was reliable method to distinguish the high salt tolerant to high salt sensitive cowpea genotypes.

\section{Conclusion}

This study provides data on the responses of cowpea genotype to salinity, which could 
be used to screen for salt tolerant parents for breeding purposes. To our knowledge, this is one of the first reports dealing with cowpea salt tolerance at germination stage which involved a large number of cowpea genotypes having a wide range of variability in terms of country of origin. The results suggest that 'PI582422', 'PI293584', 'PI582570', and '09-529' have strong salt tolerance at germination stage. In addition, the most salt-sensitive lines 'PI582813', '09393', 'PI582522', and 'PI582665' could be used as parents for developing populations for QTL mapping for cowpea salt tolerance at germination stage. Findings from this current report will add information on cowpea genotype responses to salinity at germination stage, which definitely helps breeders to select potential lines for further variety improvement process.

\section{Literature Cited}

An, P., S. Inanaga, U. Kafkafi, A. Lux, and Y. Sugimoto. 2001. Different effect of humidity on growth and salt tolerance of two soybean cultivars. Biol. Plant. 44(3):405-410.

Ashebir, G., A. Mebeasilassie, and M. Manikanidan. 2013. The response of some cowpea (Vigna unguiculata (L.) Walp.) genotypes for salt stress during germination and seedling stage. J. Stress Physiol. Biochem. 9(4):57.

Ayers, R.S. and D.W. Westcot. 1985. Water quality for agriculture. FAO irrigation and drainage paper 29. Food and Agricultural Organization, Rome.

Badia, D. and A. Meiri. 1994. Tolerance of two tomato cultivars (Lycopersicum esculentum Mill.) to soil salinity during smergence phase. Agr. Mediterr. 124:301.

Bewley, J.D. 2003. Seeds of hope; seeds of conflict. The biology of seeds. Recent Res. Adv. p. $1-10$.

Burke, J.J. 1990. High temperature stress and adaptations in crops. Plant Biol. 12:295-310.

Carter, C.T., C.M. Grieve, and J.A. Poss. 2006. Salinity effects on emergence, survival, and ion accumulation of Limonium perezii. J. Plant Nutr. 28(7):1243-1257, doi: 10.1081/PLN200063293.

Davis, D.W., E.A. Oelke, E.S. Oplinger, J.D. Doll, C.V. Hanson, D.H. Putnam, A.F. Tetchi, and G.N. Amani. 2012. Cowpea. Alternative field crops manual. University of Wisconsin Cooperative or Extension Service, Madison, WI.

Duke, J.A. 1990. Introduction to food legume, p. 1-42. In: Insect pest of tropical food legumes. John Wiley \& Sons, New York.

Dutta, P. and A.K. Bera. 2014. Effect of $\mathrm{NaCl}$ salinity on seed germination and seedling growth of mungbean cultivars. Legume Res. 37(2):161-164.

Fernandez, G.C.J. 1992. Effective selection criteria for assessing plant stress tolerance. Proc. Int. Symp. Adapt. Veg. Food Crops Temp. Water Stress. p. 257-270.

Foolad, M.R. and R.A. Jones. 1992. Parentoffspring regression estimates of heritability for salt tolerance during germination in tomato. Crop Sci. 32(2):439-442.
González, L.M. 1996. Use of radioinduction of mutations in obtaining salinity-tolerant rice genotypes. PhD Diss. (In Spanish), Granma, Cuba.

Hosseini, S.J., S. Tahmasebi, and H. Pirdashti. 2012. Analysis of tolerance indices in some rice (Oryza sativa L.) genotypes at salt stress condition. Intl. Res. J. Appl. Basic Sci. 3:1-10.

Kan, G., W. Zhang, W. Yang, D. Ma, D. Zhang, D. Hao, Z. Hu, and D. Yu. 2015. Association mapping of soybean seed germination under salt stress. Mol. Genet. Genomics 290(6):21472162.

Kent, M.L. and A. Lauchli. 1985. Germination and seedling growth of cotton: Salinity-calcium interactions. Plant Cell Environ. 8(2):155-159.

Läuchli, A. and U. Lüttge. 2002. Salinity: Environment-plants-molecules. Dordrecht, Kluwer Academic Publishers, the Netherlands.

Lobato, A.K.S., B.G. Santos Filho, R.C.L. Costa, V. Gonçalves, E.C. Moraes, C.F.O. Neto, and V.L.F. Rodrigues. 2009. Morphological, physiological and biochemical responses during germination of the cowpea (Vigna unguiculata Cv. Pitiuba) seeds under salt stress. World J. Agr. Sci. 5(5):590-596.

Maas, E.V. and G.J. Hoffman. 1977. Crop salt tolerance-current assessment. J. Irrig. Drain. Div. 103(2):115-314.

Mamiro, P.S., A.M. Mbwaga, and D.P. Mamiro. 2011. Nutritional quality and utilization of local and improved cowpea varieties in some regions in tanzania. Afr. J. Food Agr. Nutr. Dev. 11(1):4490-4506.

Mauromicale, G. and P. Licandro. 2002. Salinity and temperature effects on germination, emergence and seedling growth of globe artichoke. Agronomie 22(5):443-450.

Mistura, C., A.E.O. dos Santos, E.O. Ono, J.D. Rodrigues, M.B. de Almeida, and A.D.B. Araújo. 2011. Germination and seedlings development of the butterfly pea. Rev.Brasi. Sau. Prod. Ani. 12(2):306-317.

Muchero, W., N.N. Diop, P.R. Bhat, R.D. Fenton, S. Wanamaker, M. Pottorff, and T.J. Close. 2009. A consensus genetic map of cowpea [Vigna unguiculata (L) Walp.] and synteny based on EST-derived SNPs. Proc. Natl. Acad. Sci. USA 106(43):18159-18164.

Okiror, S.O., J.C. Onyilagha, and T. Dunbar. 2008. Investigating the potentials of four cowpea (southern pea) cultivars for fresh seed production. Intl. J. Appl. 3(1):67-74.

Olufajo, O.O. 2012. Agronomic performance of improved cowpea varieties under natural infestation with Alectra vogelii (Benth.) in the northern Guinea savannah of Nigeria. Agr. Trop. Subtrop. 45(2):66-71.

Omami, E.N. and P.S. Hammes. 2006. Interactive effects of salinity and water stress on growth, leaf water relations, and gas exchange in amaranth (Amaranthus spp.). N. Z. J. Crop Hort. Sci. 34(1):33-44.

Parker, M.B., G.J. Gascho, and T.P. Gaines. 1983. Chloride toxicity of soybeans grown on atlantic coast flatwoods soils. Agron. J. 75(3):439-443.

Pathan, M.S., J.D. Lee, J.G. Shannon, and H.T. Nguyen. 2007Advances in molecular breeding toward drought and salt tolerant crops. Springer, the Netherlands.
Reddy, K.M.M. and M.J.M. Reddy. 2016. SWOT on existing agro-ecosystem for soybean production in the northern Telangana Zone. Intl. J. Farm Sci. 6(2):268-275.

Rengasamy, P., D. Stevens, J. Kelly, M. McLaughlin, and M. Unkovich. 2006. Soil salinity and sodicity. CSIRO Publ., Collingwood, Australia.

Saad, F.F., A.A. Abd El-Mohsen, M.A. Abd, and I.H. Al-Soudan. 2014. Effective selection criteria for evaluating some barley crosses for water stress tolerance. Adv. Agr. Biol. 1(3):112-123.

Sahu, P.K. 2013. Research methodology: A guide for researchers in agricultural science, social science and other related fields. Springer, New York, NY.

Shannon, M.C. 1997. Adaptation of plants to salinity. Adv. Agron. 60:75-120.

Souza, R.P., E.C. Machado, J.A.B. Silva, A.M.M.A. Lagôa, and J.A.G. Silveira. 2004. Photosynthetic gas exchange, chlorophyll fluorescence and some associated metabolic changes in cowpea (Vigna unguiculata) during water stress and recovery. Environ. Expt. Bot. 51(1):45-56.

Stankowski, S., J.M. Sobel, and M.A. Streisfeld. 2015. The geography of divergence with gene flow facilitates multitrait adaptation and the evolution of pollinator isolation in Mimulus aurantiacus. Evolution 69(12):3054-3068.

Taffouo, V.D., L. Meguekam, M. Kenne, A. Magnitsop, A. Akoa, A. Ourry, and J.S. Tenywa. 2009. Stress effects on germination, plant growth and accumulation of metabolites in five leguminous plants. 9th African Crop Sci. Conf. Proc., Cape Town, South Africa, p. 157-161.

Tan, H., M. Tie, Q. Luo, Y. Zhu, J. Lai, and H. Li. 2012. A review of molecular makers applied in cowpea (Vigna unguiculata L. Walp.) breeding. J. Life Sci. 6:1190-1199.

TeKrony, D.M. and D.B. Egli. 1991. Relationship of seed vigor to crop yield: A review. Crop Sci. 31(3):816-822.

Thiam, M., A. Champion, D. Diouf, and M.O. Sy. 2013. $\mathrm{NaCl}$ effects on in vitro germination and growth of some senegalese cowpea (Vigna unguiculata (L.) Walp.) cultivars. ISRN Biotechnol. 2013:1-11.

Waisel, Y. 1972. Biology of halophytes. Academic Press, New York, NY.

Wallender, W.W. and K.K. Tanji. 2011. Agricultural salinity assessment and management. Am. Soc. Civ. Eng. Reston, VA.

Wests, D.W. and L.E. Francois. 1982. Effects of salinity on germination, growth and yield of cowpea. Irrig. Sci. 3(3):169-175.

Yang, J. and R.W. Blanchar. 1993. Differentiating chloride susceptibility in soybean cultivars. Agron. J. 85(4):880-885.

Zahedi, S.M., N.A. Ansari, and M. Azizi. 2012 The study of the effect of salinity stress on the germination and the initial growth of cowpea (Vigna unguiculata L. Walp). J. Agr. Technol. 8(7):2353-2372.

Zhang, X., L. Tang, C. You, and F. Hu. 2013. Effects of low temperatures on seed germination and seedling emergence of cowpea (Vigna unguiculata). J. South. Agr. 11:7. 

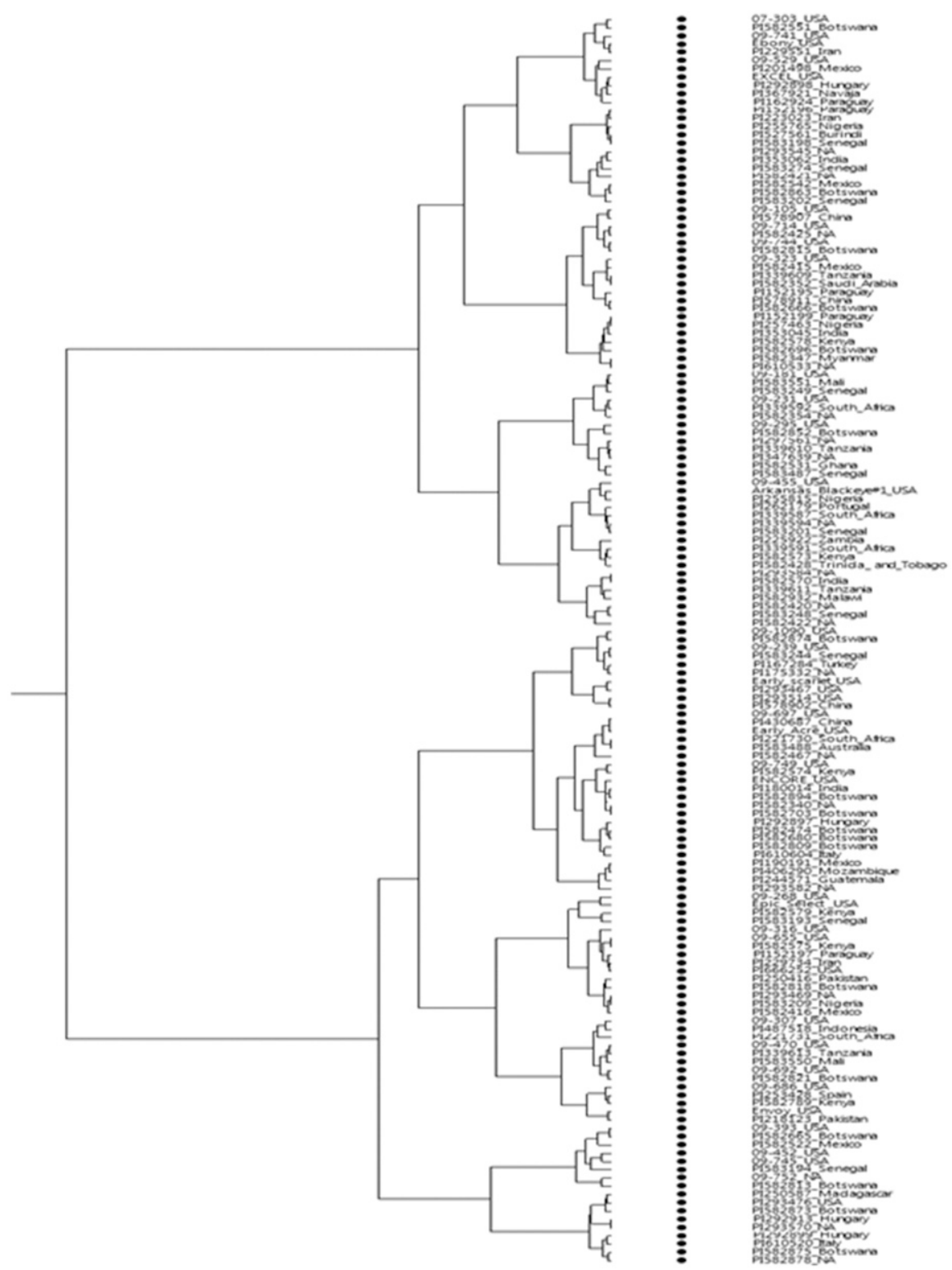

Supplemental Fig. 1. Phylogenetic tree diagram among 151 cowpea genotypes based on six salt tolerant parameters: germination rates without salt treatment (Non-stress) and under salt stress (Stress), absolute decrease (AD), inhibition index (II), relative salt tolerance (RST), and salt tolerance index (STI). 